\title{
Evaluation of Factors Impacting Cosmetic Outcome of Breast Conservative Surgery - a Study in Iran
}

\author{
Asiie Olfatbakhsh ${ }^{1 *}$, Neda Mehrdad ${ }^{1}$, Mandana Ebrahimi ${ }^{2}$, Nasrin Alavi ${ }^{1}$, Esmat \\ Hashemi $^{1}$, Ahmad Kaviani ${ }^{3}$, Masoume Najafi ${ }^{3}$, Shahpar Haghighat ${ }^{2}$, Saeed \\ Arefanian $^{5}$
}

\begin{abstract}
Background: Breast conservative surgery (BCS) followed by radiotherapy is the standard approach in management of stage I-II breast cancer. Several factors can affect cosmetic outcomes. The aim of this study was to evaluate the cosmetic results of BCS and influencing factors in the Iranian Breast Cancer Research Center. Materials and Methods: Patients who had undergone BCS were included. Photographs were taken of both breasts of the patients in three aspects and were evaluated by three specialists. The cosmetic scores were calculated based on a standard questionnaire. The data were analyzed using univariate and multivariate regression for relationships between cosmetic scores and clinical data. Results: A total number of 103 patients were included in the study. Mean age and BMI of the patients were $46.8 \pm 8.9$ and $28.1 \pm 3.9$, respectively. Breast cup sizes $C$ and $D$ accounted for $\mathbf{7 4 . 7 \%}$ of the study group. The mean cosmetic score obtained from three referees was 5.72+2.06, consisting of $35.9 \%$ excellent-good, 35\% moderate, and $29.1 \%$ unsatisfactory results. Patient BMI, volume of the resected tissue and breast cup size (D) showed significant correlation with the cosmetic score. On multivariate regression analysis, cosmetic score and BMI ( $(p=0.022$, ) as well as breast cup size $(p=0.040)$, remained significant . Conclusions: Immediate or delayed symmetrization of the breasts is suggested during breast conservative surgery, meanwhile performing oncoplastic techniques to improve the results significantly. Also it is suggested to discuss anticipation of less satisfactory results with patients having higher BMI and large breast cup size.
\end{abstract}

Keywords: Breast cancer - breast conservative surgery - cosmetic outcome

Asian Pac J Cancer Prev, 16 (6), 2203-2207

\section{Introduction}

Breast conservative surgery (BCS) followed by radiotherapy is the current standard treatment for most breast cancer cases especially in stage I-II. Although some studies have suggested that breast-conserving therapy is associated with higher local recurrence (Sun et al., 2013)

Several studies have shown that disease free survival as well as overall survival dose not differ significantly between the BCS and Modified Radical Mastectomy (MRM) (Fisher et al., 2002; Veronesi et al., 2002; Abdullah et al., 2013). Even, a recent study suggests that BCT may be associated with a higher breast cancer specific survival rate than mastectomy or mastectomy with radiation (Agarwal et al., 2014). In this point of view, one could consider BCT as the preferred modality of surgical treatment for breast cancer when eligibility criteria are fulfilled. Traditional contraindication to perform BCT includes large tumor size $(>5 \mathrm{~cm})$, skin or chest wall involvement, multicentric tumors, or anticipated poor cosmetic outcome, and whenever radiation therapy is contraindicated (Kaviani et al; 2013).

The main goal of BCS is to remove tumor with clear margin along with conserving the most possible breast tissue to achieve the best possible cosmetic outcome. Cosmetic aspects of breast surgeries has always been one of the critical concerns influencing the patient's postoperative quality of life.

Cosmetic outcome is the end result of a range of factors which come together under a broad head of surgery, radiotherapy, chemotherapy, and hormonal treatment (Munshi et al., 2009). As an instance the breast tissue would be replaced with fibrotic and fat tissue in older ages which can result poor outcomes compared to patients with younger ages. Factors most frequently reported to be important in final results are patient BMI, breast size, tumor localization, tumor size, specimen weight or volume, type of incision, chemotherapy, and irradiation (Cardoso et al., 2007). Recognizing these factors helps the surgeon to identify patients at higher risk for poor cosmetic

${ }^{1}$ Breast Diseases Research Group, ${ }^{2}$ Quality of Life Research Group, Breast Cancer Research Center, ACECR, ${ }^{3}$ Department of Surgery, Imam Khomeini Hospital, ${ }^{4}$ Department of Surgery, Cancer Institute, Tehran University of Medical Sciences, Tehran, Iran, ${ }^{5}$ Department of Surgery, Washington University in St. Louis, USA *For correspondence: Folfatbakhsh@yahoo.com 
results, discuss the probability of worse cosmetic outcome with the patient and to improve modifiable risk factors.

Most series of surgical treatment of breast cancer in Asian countries report BCT rates lower than 55\%. It is encouraging that active measures are being made in certain countries to increase BCT rates (Omranipour et al., 2014). Despite increasing numbers of breast cancer detection in early stages as well as Breast Conserving Surgeries in Iran, no evaluation of cosmetic outcomes has been reported. This cross- sectional study aimed to evaluate cosmetic results and the influencing factors of BCS procedures performed in Breast Cancer Research Center (BCRC) which works as multidisciplinary breast clinic from 1997 in Iran.

\section{Materials and Methods}

In a cross-sectional study, patients who had undergone BCS and radiotherapy at the BCRC and were followed in this center, were included in the study. Inclusion criteria consisted of 1 / having unilateral BCS with breast cancer diagnosis of stages I, II, or IIIA, 2/minimum interval time of 6 months since the last radiotherapy session, 3/ compliance to regular routine follow-up for evaluation, and 4/acceptance to obtain breast photography. Patients with metastasis or tumor recurrence were excluded from the study.

Photographs were taken by a single expert nurse from clavicle to the hypogastric region in three views: Anterior, right oblique and left oblique. All persons gave their informed consent prior to their inclusion in the study. All the photographs were evaluated by three experienced breast surgeons separately (one male and two female). The scoring system suggested by Al-Ghazal was used in this study (Amanda et al., 2006). Five factors including breast volume, breast shape, change in the nipple position, surgical scar, and radiotherapy adverse effects were considered in this assessment and each factor scored between 0 to 2 with the overall score ranging from 0 to 10 .Patients with $0-4$ score were categorized in fair result group, 5-6 score in moderate group, 7-8 in good result group and 9-10 in excellent group. Cup size of the patients was estimated based on the photography of the contralateral breast. Conventionally, investigators considered cup size A as breasts size $\leq 65$, B as 70-75, C as $80-85$, and $\mathrm{D}$ as $\geq 85$. To evaluate the resected tissue, we used the volume as it was the available data from pathologic records of the patients.

The Intra-class correlation coefficient (ICC) analysis was performed after evaluation of thirty patients by referees to confirm the consistency between them. The consistency of the assessment was considered accepTable with ICC $\geq 0.7$. Demographic, clinical, and therapeutic data of the patients were obtained from their clinical records.

The data were analyzed using SPSS-16 software. The mean and standard deviation were used for reporting data with Gaussian distribution, while median and range were used for the non-Gaussian data. The Pearson and Spearman correlation coefficient were used to describe the quantitative relationship of the studied factors. For the mean comparison of two or several factors student $t$ test and ANOVA were used, respectively. The P-value of 0.05 was considered as the statistical significance cut point.

\section{Results}

Total number of 350 patients had undergone BCS during 2001-2009 at BCRC of which 103 patients who were compliant to the follow up schedule were included. The mean age of the participants was $46.8 \pm 8.9$ (26-70) years old. Married women consisted $93 \%$ of the study group and $87 \%$ of them were gravid. About $74 \%$ of the patients were in the $\mathrm{C}$ and $\mathrm{D}$ breast cup size category.

Mean tumor size was $2.24 \mathrm{~cm} \pm 1.14$. One percent of the permanent pathologies reported positive margin and required reoperation. The mean volume of resected tissue was $271 \pm 234.4 \mathrm{cc}$. Fifty one percent of the patients were treated with Taxane-containing chemotherapy regimen and Tamoxifen was the most common used hormone therapy $(66.7 \%)$. Mean time of follow-up for the patients was $28.94 \pm 15.9$ months ranging from 6 to 95 months.

Three experienced breast surgeons evaluated breast photographs based on the five-item scoring system. The lowest score was for breast asymmetry. The mean total cosmetic score of the patients was 5.27+2.06. Overall, $35.9 \%$ of the patients were in the good-excellent category, $35 \%$ in moderate and $29.1 \%$ in the fair group (Table 1). The male surgeon reported the least scores among three referees. To evaluate the consistency of the scoring between the surgeons, the intra-class correlation coefficient test was performed and the consistency level was $0.719(0.637-0.790)$ with $95 \%$ CI which was in the accepTable range.

As presented in Table 2, analysis of variables did not show any statistically significant correlation between different clinicopathologic and therapeutic characteristics with cosmetic outcome except for patients' BMI $(\mathrm{p}=0.002)$ , Breast cup size $(\mathrm{p}=0.001)$ and volume of the resected tissue ( $\mathrm{p}=0.027$ ). The association between patient's age, tumor size, time of follow-up and number of involved lymph nodes with cosmetic outcome score were not statistically significant, although the correlation coefficient was negative implying the reverse correlation of these factors with cosmetic outcome (Not showed in Tables). Breast cup size was reversely correlated to the cosmetic outcome score implying that patients with higher cup size had lower cosmetic score. To further evaluate the relationship between cup size and cosmetic outcome, the results were analyzed by Post Hoc-Tukey test which revealed that the correlation is in fact between the breast D cup size and cosmetic score.

Variables showed significant correlation in univariate analysis were tested by multivariate linear regression test. Based on the multivariate analysis results, patients' BMI

\section{Table 1. Final Results Based on the Mean Score}

Cosmetic result Total score range Number of patients Percent

\begin{tabular}{lrll}
\hline Poor & $0-4$ & 30 & 29.1 \\
Fair & $5-6$ & 36 & 35 \\
Good & $7-8$ & 27 & 26.2 \\
Excellent & $9-10$ & 10 & 9.7 \\
\hline
\end{tabular}


Table 2. Uni-variate Analysis of Correlation between Clinico-Pathologic and Therapeutic Characteristics with Cosmetic Score

\begin{tabular}{|c|c|c|c|}
\hline Variable & Valid Percent & Mean score $\pm \mathrm{SD}$ & $P$ value \\
\hline Age at diagnosis & & & 0.48 \\
\hline Age $\leq 50$ & 63.5 & $5.82 \pm 2.055$ & \\
\hline Age $>50$ & 33.7 & $5.51 \pm 2.136$ & \\
\hline BMI & & & 0.002 \\
\hline$<25$ & 26.6 & $6.20+2.016$ & \\
\hline$>25$ & 73.4 & $5.47+2.069$ & \\
\hline Tumor Location & & & 0.448 \\
\hline Upper quadrants & 71.2 & $5.72 \pm 1.925$ & \\
\hline Lower quadrants & 18.9 & $6.12 \pm 2.053$ & \\
\hline Central & 9.9 & $5.10+3.027$ & \\
\hline Tumor grade & & & 0.712 \\
\hline $\mathrm{I}$ & 12.0 & $6.15 \pm 1.237$ & \\
\hline II & 64.0 & $5.53 \pm 1.948$ & \\
\hline III & 24.0 & $5.54 \pm 2.696$ & \\
\hline Stage & & & 0.737 \\
\hline 0 & 7.2 & $6.14 \pm 3.495$ & \\
\hline 1 & 16.5 & $5.60 \pm 2.056$ & \\
\hline 2 & 53.6 & $5.83 \pm 1.923$ & \\
\hline 3 & 22.7 & $5.32 \pm 2.064$ & \\
\hline Axillary Lymph Nodes & & & 0.609 \\
\hline Negative & 47.4 & $5.83 \pm 2.153$ & \\
\hline Positive & 52.6 & $5.61 \pm 2.040$ & \\
\hline Tumor Size & & & 0.702 \\
\hline$\leq 2 \mathrm{~cm}$ & 49.0 & $6.03 \pm 2.103$ & \\
\hline$>2 \mathrm{~cm}$ & 51.0 & $5.39 \pm 2.064$ & \\
\hline Breast Cup Size & & & 0.001 \\
\hline A & 2.9 & $7.67 \pm 0.882$ & \\
\hline B & 22.1 & $6.52 \pm 1.445$ & \\
\hline $\mathrm{C}$ & 49.0 & $5.86 \pm 2.248$ & \\
\hline $\mathrm{D}$ & 25.0 & $4.49 \pm 1.620$ & \\
\hline Axillary Incision & & & 0.156 \\
\hline separate & 58.2 & $5.88 \pm 2.1777$ & \\
\hline Accompanied with breast incision & 41.8 & $1.905 \pm 1.905$ & \\
\hline Type of incision & & & 0.322 \\
\hline Radial & 52.5 & $5.53 \pm 2.196$ & \\
\hline Curvilinear & 16.8 & $5.43 \pm 1.747$ & \\
\hline Peri-areolar & 30.7 & $6.18 \pm 2.020$ & \\
\hline Chemotherapy & & & 0.520 \\
\hline No Chemotherapy & 12.5 & $6.50 \pm 2.834$ & \\
\hline Adriamycin- containing & 30.2 & $5.69 \pm 2.197$ & \\
\hline Taxane-containing & 51.0 & $5.68 \pm 1.790$ & \\
\hline Other regiments & 2.0 & $5.11 \pm 2.335$ & \\
\hline Volume of resected tissue & & & 0.027 \\
\hline$<200 \mathrm{cc}$ & 51.8 & & \\
\hline$>200 \mathrm{cc}$ & 48.2 & & \\
\hline
\end{tabular}

Table 3. Multi-Variate Analysis of the Factors Affecting Cosmetic Score

\begin{tabular}{lccc}
\hline Parameters & B & S.E. & P Value \\
\hline BMI & -0.128 & 0.055 & 0.022 \\
Volume of resected tissue & -0.002 & 0.001 & 0.74 \\
Breast cup size & -1.040 & 0.499 & 0.040 \\
Constant & 9.973 & 1.524 & $<0.001$ \\
\hline
\end{tabular}

and breast D cup size were the factors that influence the cosmetic outcome of BCS independently (Table 3).

\section{Discussion}

The objective of the present study was to evaluate the cosmetic results of breast conserving surgeries and to characterize factors influencing them. The incidence of patients with good or excellent cosmetic result in this series was lower than similar studies and the most important risk factors were large breast cup size and higher BMI.

In this study, the cosmetic results were evaluated by panel. Care should be used in interpreting results where cosmetic outcomes have been obtained from a single evaluator.

Different methods have been suggested to evaluate cosmetic outcome of breast surgery with controversy for deciding the most comprehensive one. The most popular used criteria are suggested by Harris et al (Harris et al., 1979): i) Excellent: the treated breast almost identical to the untreated one. ii) Good: the treated breast slightly 
different from the untreated breast. iii) Fair: obvious difference between the two sides without major distortion. iv) Poor: the treated breast is seriously distorted.

However in more detailed assessment systems like Al-Ghazal multi-item scoring system which is used in this study, cosmetic features could be scored which include: shape and volume symmetry, scar quality and skin changes. The multi-item scales can provide more information with regards to what specifically is wrong with the cosmetic appearance of the breast and should be corrected. (Amanda et al., 2006)

Overall Cosmetic scores in this study compared to the other similar study in Iran are lower (Kaviani et al., 2010) (5.72 of 10 compared to 9 of 14). The difference in the used scoring system can be considered as one of the main reasons. Another important reason for higher cosmetic score in Kaviani's report would be that the investigators merely included data of oncoplastic breast surgeries which uses simultaneous breast tissue reconstructive techniques compared to our study that covers BCS overall without summarization. Also our data reported lower score compared to some similar published international studies with a rate of excellent and good proportion ranging from $52.9 \%$ to $89.8 \%$ (Deutsch et al., 2003; Arenas et al., 2006; Schultze et al., 2008). Meanwhile it should be considered that in some of them such as Deutsch et al, high cosmetic score was the result of a single referee evaluation (Deutsch et al., 2003).

Our study showed that the lowest scores belonged to the asymmetric breast volume compared to the contralateral breast followed by breast shape. The high proportion of patients with $\mathrm{C}$ and $\mathrm{D}$ breast cup sizes might be the most reason of breast asymmetry.

In this study three referees scored the breast photographs with the male oncoplastic surgeon having the lowest reported scores, a result similar to Fortin et al study (2006). The mean age of the patients in the present study was $46.8 \pm 8.9$ with $63.6 \%$ less than 50 years which was consistent with the similar national study, however it was lower compared to the international studies. In a study performed by Johansen et al the mean of patients' age was 50 (Deutsch et al., 2003), while this value in the study published by Deutsch et al was 61years old with $25.3 \%$ being younger than 50 (Johansen et al., 2002).

About three forth $(74.7 \%)$ of the patients in our study had breast $\mathrm{C}$ and $\mathrm{D}$ cup sizes implying their high breast volumes which resulted in the lower score for their asymmetry after the surgery compared to the contralateral breast. High breast size is a commonly mentioned factor with negative effect on the surgery (Harris et al., 1979; AlGhazal et al., 1999; Cardoso et al., 2007). Tumor location did not show a significant correlation with cosmetic results, similar to the results reported by Clarke and Rose (Clarke et al., 1983; Rose et al., 1989), but Sacchini et al mentioned that tumors located in the lateral breast region have better results (Sacchini et al., 1991). Despite the controversy regarding the correlation of patient age and cosmetic outcome, most of the studies have reported better results with lower patient age, a correlation that could not reach statistical significance in our study. $(\mathrm{p}=0.48)$ (Taylor et al., 1995).
The univariate analysis of this study showed that BMI, breast size, and resected tissue volume were significantly effective on the cosmetic results. Clarke et al noted a correlation between body weight and cosmetic results, with patients less than $55 \mathrm{~kg}$ having excellent cosmesis in $90 \%$, whereas a group weighing more than $73 \mathrm{~kg}$ had only $46 \%$ excellent outcomes (Clarke et al., 1983). This latter finding may also be related to breast size because heavier women have usually larger breasts with more fat.

As we noted, the lowest cosmetic score between five items of scoring system in this study was for asymmetry and in large breasts difference in breasts' size would be more distinguishable. The worst outcome was for patients with cup size $\mathrm{D}$ after multivariate analysis which approve other studies (Harris et al., 1979).

Volume of the resected breast tissue had a direct impact on cosmesis. Only isolated small studies have not shown any correlation between extent of surgical excision and cosmesis (Touboul et al., 1995). Olivotto et al suggested that tumors greater than $70 \mathrm{~cm} 3$ result in a significant increase in the number of cosmetic failures (Amanda et al., 2006). Garofalo et al overcome this problem by performing a contralateral quadrantectomy to achieve symmetry of both sides (Garofalo ,et al.,1992).

Beside the common studied factors, correlation of other probable factors such as marital status, pregnancy history, side of the operated breast, hormone therapy regimen, chemotherapy regimen, as well as axillary incision type with cosmetic score were evaluated in the study, but no significant correlation was found. In spite of our results, in a study with 6.6 years follow up of 266 patients, Johansen et al found performing chemotherapy with $\mathrm{CMF}$ regimen and radiotherapy as two more effective factors on cosmetic results (Johansen et al., 2002).

In conclusion, the results of this study showed that volume of the resected tissue, BMI, and breast cup size have correlation with lower cosmetic outcome in breast conservative surgery. Within these factors BMI and breast size are impossible or hard to change, but it should be mentioned to patients that their chance of getting satisfactory results may be not as good as usual and consider symmetrization for the contralateral breast.

Volume of the resected tissue is the single modifiable factor. Attending balance between this factor and oncologically safe margin is the main challenge for surgeons during BCS. Applying Oncoplastic breast surgery (OBS) that provide both of these aspects is an answer to this problem. Different techniques of OBS have been introduced in recent years, the investigations on aesthetic outcomes after OBS are very few. According to these few studies it seems that there are some important advantages for OBS in comparison with the other traditional surgeries.

\section{References}

Agarwal S, Pappas L, Neumayer L, et al (2014). Effect of breast conservation therapy vs mastectomy on disease-specific survival for early-stage breast cancer. JAMA Surg, 149, 267-74.

Abdullah A, Abdullah KL, Yip CH, et al (2013). The decisionmaking journey of Malaysian women with early breast 
cancer: A qualitative study. Asian Pac J Cancer Prev, 14, 7143-7.

Fortin AJ, Cheang M, Latosinsky S (2006). Cosmetic outcomes following breast conservation therapy: in search of a reliable scale. Breast Cancer Res treat, 100, 65-70.

Arenas M, Sabater S, Hernandez V, et al.(2006). Cosmetic outcome of breast conservative treatment for early stage breast cancer. Clin Transl Oncol, 8, 334-8.

Al-Ghazal SK, Fallowfield L, Blamey RW (1999). Patient evaluation of cosmetic outcome after conserving surgery for treatment of primary breast cancer. Eur J Surg Oncol, 25, 344-6.

Al-Ghazal SK, Blamey RW (1999). Cosmetic assessment of breast-conserving surgery for primary breast cancer. Breast, 8, 162-8.

Cardoso MJ, Cardoso J, Santos AC, et al (2007). Factors determining esthetic outcome after breast cancer conservative treatment. Breast J, 13, 140-6.

Clarke D, Martinez A, Cox RS (1983). Analysis of cosmetic results and complications in patients with stage I and II breast cancer treated by biopsy and irradiation. Int J Radiat Oncol Biol Phys, 9, 1807-13.

Deutsch M, Flickinger JC (2003). Patient characteristics and treatment factors affecting cosmesis following lumpectomy and breast irradiation. Am J Clin Oncol, 26, 350-3.

Fortin AJ, Cheang M, Latosinsky S (2006). Cosmetic outcomes following breast conservation therapy: in search of a reliable scale. Breast Cancer Res Treat, 100, 65-70.

Fisher B, Anderson S, Bryant J, et al (2002). Twenty-year follow-up of a randomized trial comparing total mastectomy, lumpectomy, and lumpectomy plus irradiation for the treatment of invasive breast cancer. $N$ Engl J Med, $\mathbf{3 4 7}$, 1233-41.

Garofalo R, Borioni R, Garofalo RLL, et al (1992). Radical tumor excision and cosmetic balance in the surgical treatment of breast carcinoma: biquadrantectomy. Biomed Pharmacother, 46, 401-4.

Harris JR, Levene MB, Svensson G, Hellman S (1999). Analysis of cosmetic results following primary radiation therapy for stages I and II carcinoma of the breast. Int J Radiat Oncol Biol Phys, 5, 257-61.

Johansen J, Overgaard J, Rose C, et al (2002). Cosmetic outcome and breast morbidity in breast-conserving treatment--results from the Danish DBCG-82TM national randomized trial in breast cancer. Acta Oncol, 41, 369-80.

Kaviani A,Taslimi Sh, Athari B, et al (2010). The cosmetic results after oncoplastic breast surgery in Iranian women. Tehran University Med J, 68, 213-9.

Kaviani A, Sodagari N, Sheikhbahaei S, et al (2013). From radical mastectomy to breast-conserving therapy and oncoplastic breast surgery: a narrative review comparing oncological result, cosmetic outcome, quality of life, and health economy. ISRN Oncol, 2013, 742462

Liauw, S.H., L.J. Sanfilippo, E. Santoro,(1987). Breast size versus cosmesis and local control in stages I and II breast carcinoma. $N$ Engl J Med, 84, 706-10.

Munshi, S. Kakkar, R. Bhutani, R. et al (2009). Factors influencing cosmetic outcome in breast conservation. Clin Oncol, 21, 285-93,

Omranipour R, Alipour S, Hadji M, et al (2014). Two decades of experience with ductal carcinoma in situ of the breast in the Cancer Institute of Tehran, Iran. Asia Pac J Cancer Prev, 15, 2771-6

Rose MA, Olivotto I, Cady B, et al (1989). Conservative surgery and radiation therapy for early breast cancer. Long-term cosmetic results. Arch Surg, 124, 153-7.

Sun M-Q, Meng A-F, Huang X-E, et al (2013). Comparison of psychological influence on breast cancer patients between breast-conserving surgery and modified radical mastectomy. Asian Pac J Cancer Prev, 14, 149-52.

Schultze J, Lossl K, Kimmig B (2008). [Cosmetic results after breast conserving carcinoma treatment in patients with intramammarian seromas]. Rontgenpraxis, 56, 169-80.

Sacchini 1, Luini A, Tana S et al (1991). Quantitative and qualitative cosmetic evaluation after conservative treatment for breast cancer. Eur J Cancer, 27, 1395-400.

Tan MP-T, Sitoh NY-Y, Sim AS-T (2014) Evaluation of eligibility and utilization of breast conservation treatment in an Asian context. Asian Pac J Cancer Prev, 15, 4683-4688

Taylor ME, Perez CA, Halverson KJ, et al (1995). Factors influencing cosmetic results after conservation therapy for breast cancer. Int J Radiat Oncol Biol Phys, 31, 753-64.

Touboul E, Belkacemi Y, Lefranc JP, et al. (1995). Early breast cancer:influence of type of boost (electrons vs iridium-192 implant) on local control and cosmesis after conservative surgery and radiation therapy. Radiother Oncol,34, 105-13.

Veronesi U, Cascinelli N, Mariani L et al (2002). Twentyyear follow-up of a randomized study comparing breastconserving surgery with radical mastectomy for early breast cancer. N Engl J Med, 347, 1227-32. 